\title{
Wafer Level Packaged CMOS-SOI-MEMS Thermal Sensor at Wide Pressure Range for IoT Applications ${ }^{\dagger}$
}

\author{
Moshe Avraham 1, Gady Golan 1, Michele Vaiana 2, Giuseppe Bruno 2, Maria Eloisa Castagna 2, \\ Sara Stolyarova ${ }^{3}$, Tanya Blank ${ }^{3}$ and Yael Nemirovsky ${ }^{3, *}$ \\ 1 Ariel University, Ariel 40700, Israel; email1@gmail.com (M.A.); gadigolan@gmail.com (G.G.) \\ 2 STMicroelectronics, Stradale Primosole, 50-95121 Catania, Italy; email2@gmail.com (M.V.); \\ email3@gmail.com (G.B.); email4@gmail.com (M.E.C.) \\ 3 Electrical Engineering Dept., Technion-Israel Institute of Technology, Haifa 32000, Israel; \\ email5@gmail.com (S.S.); email6@gmail.com (T.B.) \\ * Correspondence: nemirov@technion.ac.il \\ + Presented at the 7th Electronic Conference on Sensors and Applications, 15-30 November 2020; \\ Available online: https://ecsa-7.sciforum.net/.
}

Published: 15 November 2020

\begin{abstract}
Wafer Level Processed and Wafer Level Packaged low-cost MEMS (Micro Electro Mechanical Systems) thermal sensors are required for a wide range of IoT and wearables applications. Recently, a new generation of uncooled thermal sensors based on CMOS-SOI-MEMS technology has emerged, with higher performance compared to commercial thermal sensors (bolometers, thermopiles, and pyroelectric sensors). The technology is implemented in commercial CMOS FABs and is therefore based on mature technology and implemented at a low cost. When packaged in a high vacuum, the sensors are dubbed TMOS and are applied for uncooled IR radiation. At atmospheric pressure, the sensors may function as Gas Sensors, dubbed GMOS. This paper focuses on the study of thermal performance of wafer level processed and packaged TMOS and GMOS sensors, where the pressure varies between high vacuum $(0.01 \mathrm{~Pa})$ and atmospheric pressure. The present study is based on analytical thermal modeling for gaining physical insight, 3D simulation is performed by ANSYS software and finally, the measurements of packaged devices are compared with the modeling and simulations.
\end{abstract}

Keywords: TMOS; thermal uncooled IR sensors; wearables; CMOS-SOI-MEMS; wafer-level packaging; thermal performance of radiation and gas sensors; FEA simulations; ANSYS; IOT

\section{Introduction}

Thermal IR sensors are widely used in various fields such as automotive, IoT applications, human intruder alert systems as well as smart building management (lighting, heating) or temperature sensing, with an increasing market forecast for the next years. Commercial technologies currently in use for uncooled infrared devices are bolometers, thermopiles, and pyroelectric sensors. More recently, a new generation of uncooled thermal sensors based on CMOS-SOI-MEMS technology has emerged, dubbed "TMOS" [1].

The TMOS is a MEMS (Micro Electro Mechanical Systems) device based on a suspended, thermally isolated micro-machined floating transistor, which absorbs infrared radiation. The resulting temperature change is transduced into an electric signal. The TMOS operates at the subthreshold region, therefore requiring low power consumption, which is essential for wearable applications. Moreover, the inherent gain of the transistor results in the highest temperature sensitivity, compared to the commercial thermal sensors [2]. By adding a heated catalytic layer above 
the TMOS thermal sensor, a highly sensitive chemical gas sensor, dubbed GMOS, has been reported [3-5]. The TMOS highest performance is achieved in a high vacuum whereas the GMOS is operated at ambient pressure. Regardless of the application, like any MEMS device, the TMOS as well as the GMOS must be packaged to protect the delicate structure from dust and particles as well as to achieve the optimal performance. Wafer Level Processing as well as Wafer Level Packaging with a controlled vacuum is essential to ensure high performance and low cost $[6,7]$.

This paper focuses on the study of thermal performance of a wafer level processed and packaged TMOS where the pressure varies between high vacuum $(0.01 \mathrm{~Pa})$ and atmospheric pressure (Section 2). The present study is based on analytical thermal modeling for gaining physical insight [8] (Section 3). In the presence of air or other gas, a 3D simulation is mandatory since there is a significant thermal conductance by the gas surrounding the TMOS stage in the package. Finite Element Analysis (FEA) simulations are performed by ANSYS software [9] (Section 4). Finally, the measurements of packaged devices are compared with the modeling and simulations [10] (Section 5).

\section{Wafer Level Processing (WLP) and Wafer Level Packaging (WLP) of the TMOS Sensor [7]}

The TMOS sensors are fabricated in a standard CMOS-SOI FAB, while the metal layers provide built-in masks for the MEMS processing. The back-side handle is removed by DRIE the BOX layer providing etch stop. Front-side dielectric layers are removed by RIE while the front-side metal masks are removed by etching. With this method, a thermally insulated transistor is achieved. The arm configuration and the TMOS dimensions are optimized for specific applications. In order to increase the thermal insulation, the interconnecting wires on the holding arms are made of active-silicon and polysilicon instead of metals. The CMOS-SOI-MEMS unique technology provides good alignment that is determined by the CMOS-SOI technology (130 nm in the present study), and there is no need for expensive masks.

In order to encapsulate the TMOS sensor, a WLP has been developed [7]. The WLP provides the required high vacuum and long-term hermetic package for the TMOS performance and stability. The top silicon cap and bottom silicon cap are bonded to the silicon wafer with a glass-frit bonding technique. Glass frit has a low melting point, under $450[\mathrm{~K}]$, and hence is compatible with the CMOSSOI technology. In the bonding process, the heated melted glass and compression are applied to the wafer and after cooling the glass solidifies and creates a high bond strength between the wafer and the silicon caps encapsulating the TMOS at low pressure. A gettering layer is added to the bottom cap in order to remove residual gases. Figure 1 presents schematically a single TMOS sensor pixel and a WLP TMOS sensor array.

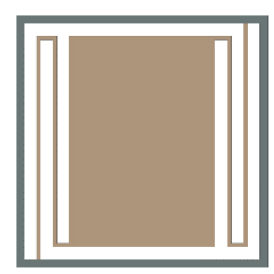

(a)

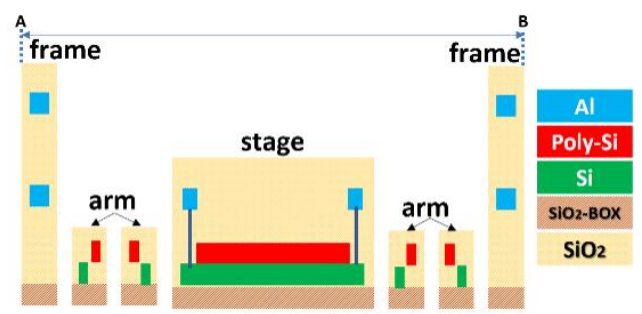

(b)



(c)

Figure 1. Schematic TMOS sensor (a) top view; (b) cross-section of a single TMOS pixel; (c) crosssection of WLP TMOS sensor array.

\section{Thermal Modeling}

The operation principle of the TMOS, as a thermal IR sensor, is straightforward: a radiation power absorbed in the thermally insulated sensor stage causes a temperature change. The TMOS transistor transduces the temperature change into an electric signal: current or voltage. The power balance equation is expressed by:

$$
\eta P_{\text {opt }}=C_{t h}(d(\Delta T(t)) / d t)+G_{t h} \Delta T(t)
$$


where $\eta$ is the absorption efficiency, $C_{t h}$ is the thermal capacitance, $G_{t h}$ is the thermal conductance of the system. From the steady-state solution of (1), the relation between the temperature change of the TMOS to the system thermal conductance is given by:

$$
\Delta T_{s s}=\eta P_{\text {opt }} / G_{t h}
$$

The WLP TMOS model is designed by the lumped circuit assumption that the heat conductance is governed by the arm only (at very low pressure) or by the packaged gas (at medium or high pressure) as will be shown. The thermal capacitance is governed by the TMOS stage only, and is independent of the pressure. For convenience, the electric analog property is compared to the thermal one in Table 1 and the model operation is represented in Figure 3.

Table 1. Analog thermal domain properties to electric domain properties.

\begin{tabular}{l|c|l|c}
\hline \multicolumn{2}{c|}{ Flectric property } & \multicolumn{2}{c}{ Thermal property } \\
\hline Conductance & $G\left[\Omega^{-1}\right]$ & Conductance & $G_{t h}[W / K]$ \\
Capacitance & $C[F]$ & Capacitance & $C_{t h}[J / K]$ \\
Voltage & $V[V]$ & Temperature & $T[K]$ \\
Charge & $q[C]$ & Heat & $Q[J]$ \\
Current & $i[A]$ & Heat density & $P[W]$ \\
Time constant & $\tau[s]$ & Time constant & $\tau_{l h}[s]$ \\
\hline
\end{tabular}

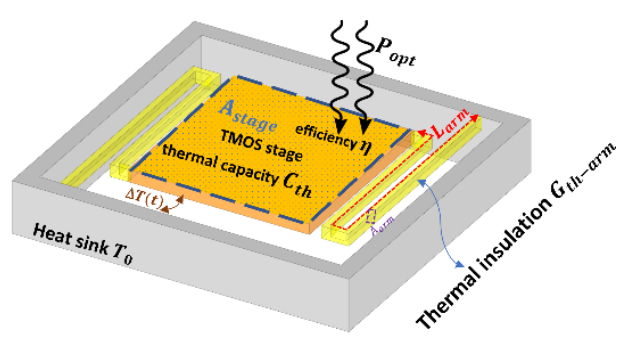

(a)

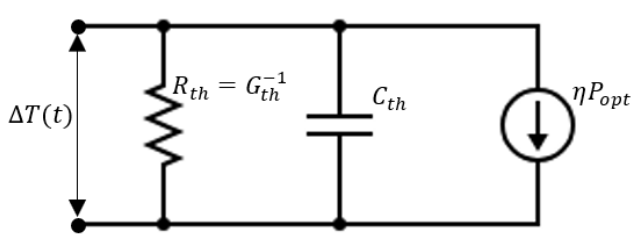

(b)

Figure 3. Model operation of the TMOS (a) schematic; (b) equivalent electric network.

The system thermal capacitance is not varying with the pressure and equals to:

$$
C_{\text {th }}=\rho c^{\prime} A_{\text {stage }} t
$$

where $\rho$ is the material mass density, $c^{\prime}$ is the specific heat capacity, $A_{\text {stage }}$ is the TMOS stage area and $t$ is the stage thickness. The thermal time constant of the sensor is defined by:

$$
\tau_{t h}=C_{t h} / G_{t h}
$$

The thermal time constant may be measured experimentally (see Section 5). The thermal conductance is determined by the thermal conductance of the solid elements, the thermal conductance of the gas, and thermal conductance by radiation. The thermal conductance of the solid is independent of the pressure, and as mentioned at high vacuum is governed by the holding arm (see Figures1 and 2) and equal to:

$$
G_{\text {arm }}=k A_{\text {arm }} / L_{\text {arm }}
$$

where $k$ is the thermal conductivity of the material, $A_{\text {arm }}$ is the arm area that the heat flows through and $L_{\text {arm }}$ is the arm length as marked in Figure 3a.

In the case of a high vacuum, where the heat conduction occurs by the solid holding arm, the thermal properties of the packaged TMOS can be calculated analytically [8]. In the presence of air or other gas, a 3D simulation is mandatory since there is a significant thermal conductance by the gas surrounding the TMOS stage in the package. Hence in the presence of gas, the total thermal conductance is the serial combined thermal conductance of the arm, gas, and radiation:

$$
G_{\text {th }}=G_{\text {arm }}+G_{\text {gas }}+G_{\text {rad }}
$$

The radiation power of a black body is known and is given by Stefan-Boltzmann law: 


$$
P_{\text {rad }}=\varepsilon \sigma A_{\text {stage }} T_{s}^{4}
$$

where $\varepsilon$ is the body emissivity, $\sigma$ is Stephan-Boltzmann constant. Therefore, the thermal conductance by radiation is given by:

$$
G_{\text {rad }}=d P_{\text {rad }} / d T=4 \varepsilon \sigma A_{\text {stage }} T_{s}^{3}
$$

The gas thermal conductance is strongly dependent on the pressure, in contrast to the thermal conductance of the solid holding arm. In order to perform simulations of the 3D WLP TMOS sensor for wide pressure range, with FEA software (Section 4), the thermal conductivity, $k$, of the gas should be known. For atmospheric pressure, the thermal conductivity is well established and has been found to lie between $0.02-0.03[\mathrm{~W} / \mathrm{mK}]$, for many common gases like oxygen at normal ambient conditions $\left(1[\mathrm{~atm}],{ }^{\circ} \mathrm{C}\right)[8]$.

\subsection{Modelling Gas at High Pressure (around Ambient Pressure)}

For dense, high-pressure gas, the heat flow between two plates separated by a distance $d$, can be expressed by [11]:

$$
G_{\text {high-pressure }}^{\prime \prime}=k / d\left[\mathrm{~W} / \mathrm{K} \cdot \mathrm{m}^{2}\right]
$$

At high pressure, the mean free path of the gas molecules is small, because it is governed by the molecules collisions to themselves and the value of the thermal conductivity is dependent only on the temperature. For air, it is well known and established to be $k_{\text {high-pressure }}=0.026[\mathrm{~W} / \mathrm{m} \cdot \mathrm{K}]$ at $300[\mathrm{~K}]$.

\subsection{Modelling Gas at Low Pressure (Where Mean-Free-Path Is Large)}

At very low pressures where the mean-free-path becomes much larger than the distance between plates, a molecule can transfer heat directly from one plate to another. In this case, the heat flow is proportional to the number of molecules and the temperature differences between the plates. Thus, at low pressure, the heat flow can be expressed by [11]:

$$
G_{\text {low-pressure }}^{\prime \prime}=G_{0}^{\prime \prime} \cdot \frac{p}{p_{0}}=\frac{1}{3} \rho C_{v} v_{m o l}\left[\frac{\mathrm{W}}{\mathrm{K} \cdot \mathrm{m}^{2}}\right]
$$

where $\rho$ is the gas density, $C_{v}$ is the gas specific thermal capacity and $v_{m o l}$ is the average thermal velocity of the molecule. The thermal velocity is equal to $\sqrt{3 k_{B} T / m}$, where $m$ is the molecule mass and $k_{B}$ is Boltzman constant. The value of the thermal velocity for air at $1[\mathrm{~Pa}]$ and $300[\mathrm{~K}]$ is about $500[\mathrm{~m} / \mathrm{s}]$. The density is $1.16 \cdot 10^{-5}\left[\mathrm{~kg} / \mathrm{m}^{3}\right]$ and the specific heat is $1007\left[\mathrm{~J} / \mathrm{K} \cdot \mathrm{m}^{2}\right]$, hence for these values the heat flow is $\sim 2\left[\mathrm{~W} / \mathrm{K} \cdot \mathrm{m}^{2}\right]$.

The relation between the thermal conductivity to the heat flow:

$$
k=G^{\prime \prime} \cdot l
$$

where $l$ is equal to the mean free path, which in this study is approximately equal to the smallest gap in the device, because the probability the molecules collide by the gap distance (3 [um]) is much larger than the probability the molecules collide by the plate distance (150 [um]) or by the molecules to themselves.

\subsection{Modelling Gas at Intermediate Pressures}

At intermediate pressures, where the mean free path is larger by a factor of approximately one hundred compared to the separating distance between the plates $d$, the heat flow by the gas occurs by both mechanisms. Consequently, the heat flow by the gas that causes the total temperature difference between the plates is equal to the serial combination of the two mechanisms [8]:

$$
1 / k_{\text {gas }}=1 / k_{\text {high-pressure }}+1 / k_{\text {low-pressure }}
$$


From (10)-(12) the thermal conductivity, $k_{\text {air }}$ can be extracted for any pressure range. Figure 4 shows the thermal conductivity of air, for a wide pressure range. The thermal conductivity of the air is a parallel combination of the thermal conductivity at high pressure with the thermal conductivity at low pressure.

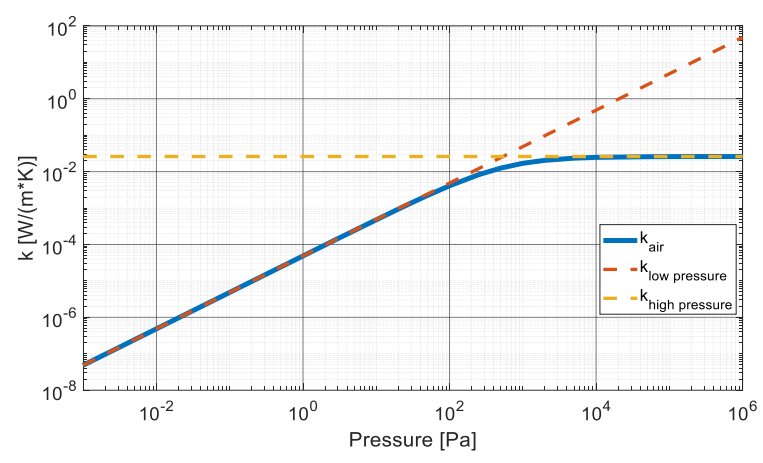

Figure 4. Thermal conductivity of the air vs. the pressure.

The drawback of this modeling method is the fact that at low and intermediate pressure the thermal conductivity is not constant. If the mean free path is larger than the distance of the plates, this is problematic since two different surfaces at different distances require two different thermal conductivities in the same simulation. But taking into consideration that the total thermal conductivity is parallelly combined, the smallest value becomes the most significant one and thus the total thermal conductivity is governed by the smallest typical gap of the MEMS structure.

\section{Simulations and Results}

A 3D model of $4 \times 4$ TMOS sensor WLP array has been modeled using a commercial FEA software. The material's thermal properties of the solid elements were provided by the FAB (please refer to Table 2). The modeled gas inside the package is air, the specific heat capacity is typically independent of pressure and the assumed value is $1007[\mathrm{~J} / \mathrm{kg} \cdot \mathrm{K}]$. The density was defined by the ideal gas law as a function of pressure and the thermal conductivity was calculated as described in Section 3.

Table 2. Thermal properties of CMOS-SOI process.

\begin{tabular}{ccccc}
\hline Thermal properties & SiO2 & Poly Si & Si & Al \\
\hline Thermal Conductivity, $k[W /(\mathrm{m} \cdot \mathrm{K})]$ & 1.4 & 40 & 40 & 201 \\
Specific Heat Capacity, $\mathrm{Cp}[\mathrm{J} /(\mathrm{kg} \cdot \mathrm{K})]$ & 730 & 678 & 700 & 900 \\
Density, $\rho\left[\mathrm{kg} / \mathrm{m}^{3}\right]$ & 2200 & 2320 & 2329 & 2700 \\
\hline
\end{tabular}

Thermal boundary conditions such as the outside of the WLP sensor remain at constant temperature and are set assuming uniform heat power of $1 \mathrm{uW}$ on each TMOS pixel stage. The thermal conductance of the solids and the gas of the TMOS WLP can be evaluated from a steady-state thermal simulation that yields the final temperature of the device, with (2). Figure 5 shows the temperature results of the simulation for high pressure $(\sim 1 \mathrm{~atm})$ and for low pressure $(2.5 \mathrm{~Pa})$. The average temperature difference of the TMOS stage is $0.15[\mathrm{~K}]$ and $7.5[\mathrm{~K}]$, respectively. Since the transistor, operating at subthreshold transduces the temperature differences into an electric measured signal, this illustrates the tremendous effect of the packaged gas pressure on the sensor performance. For low pressures the model assumptions are valid, since the stage temperature distribution is approximately uniform, as shown below in Figure 5a. 


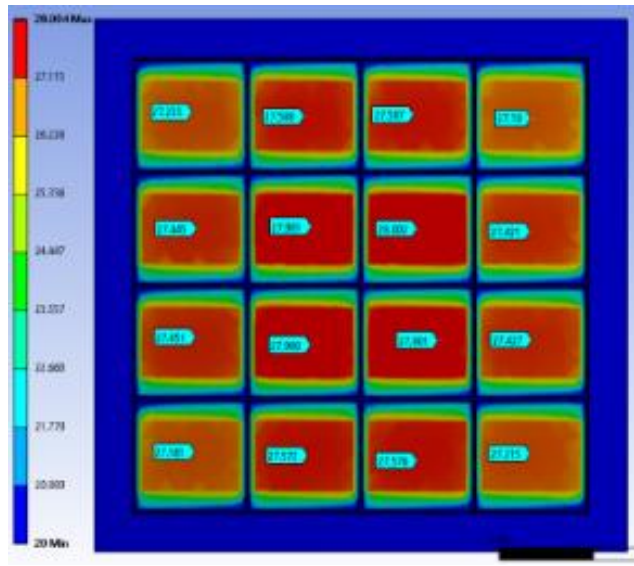

(a)

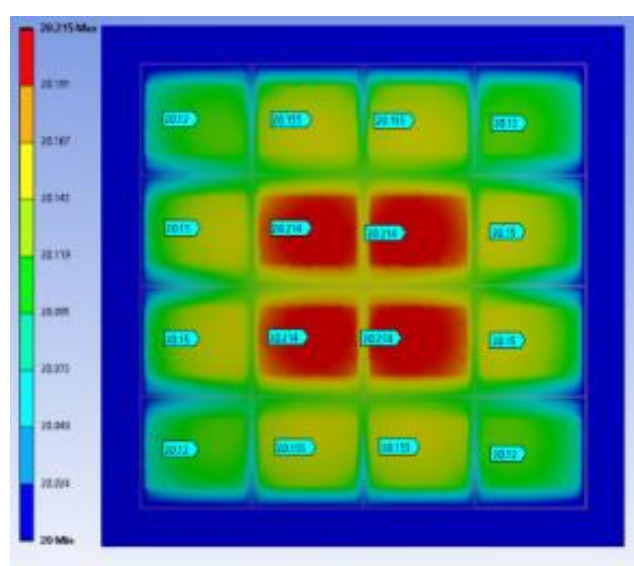

(b)

Figure 5. Steady-state simulation results of $4 \times 4$ WLP TMOS sensor array: (a) for air pressure $2.5[\mathrm{~Pa}]$, the color bar represents temperature from $20\left[{ }^{\circ} \mathrm{C}\right]$-blue to $28\left[{ }^{\circ} \mathrm{C}\right]$-red; (b) for air pressure $10^{5}[\mathrm{~Pa}]$, the color bar represents temperature from $20\left[{ }^{\circ} \mathrm{C}\right]$-blue to $20.215\left[{ }^{\circ} \mathrm{C}\right]$-red.

After receiving the thermal conductance by the gas and solids from the simulation results, the total thermal conductance of the system, including radiation can be evaluated by solving equations $(6,8)$ iteratively. The results are shown in Figure 6.

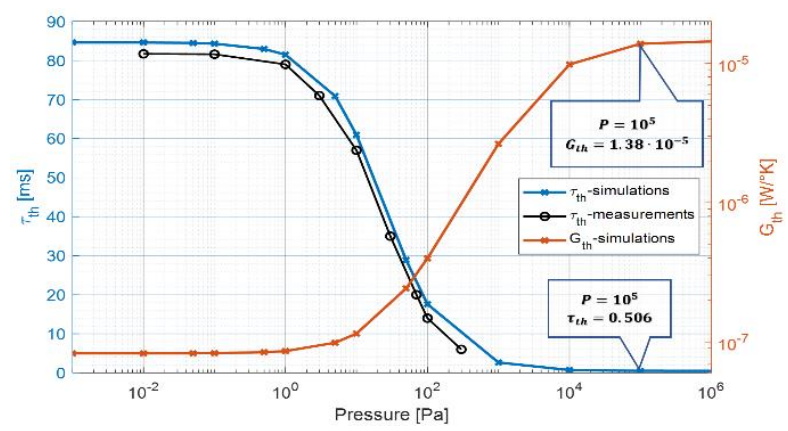

Figure 6. Wide pressure range simulation and measurement results of the system thermal time constant and the simulation results of the system thermal conductance as a function of the pressure.

The thermal conductance is changing from $8.3 \cdot 10^{-8}[\mathrm{~W} / \mathrm{K}]$ at low pressure $10^{-3}-10^{-2}[\mathrm{~Pa}]$ to $1.45 \cdot 10^{-5}[\mathrm{~W} / \mathrm{K}]$ at atmospheric pressure $10^{5}[\mathrm{~Pa}]$. It should be noted that at a high vacuum where heat is transferred by conduction through the holding arms, the analytically modeled $G_{t h}$ and the simulated $G_{t h}$ are in good agreement. At atmospheric pressure, the main heat transfer mechanism is governed by the gas. By applying the $k$ of the gas at atmospheric pressure, the distance $d$ to the top packaging filter, and the total area of the 16 suspended, micromachined plates, the analytically evaluated $G_{t h}$ agrees with the simulated one. The thermal time constant is changing from 84.6 [ms] at low pressure $10^{-3}-10^{-2}[\mathrm{~Pa}]$ to $0.5[\mathrm{~ms}]$ at atmospheric pressure $10^{5}[\mathrm{~Pa}]$.

\section{Measurements}

A methodology of measuring the pressure inside a tiny MEMS device was developed [10]. The experiment was performed to measure the thermal time constant in a TMOS sensor by varying the pressure inside the cavity in a controlled environment and detaily described in [7]. The thermal time constant was $\cong 81[\mathrm{~ms}]$ at low pressure $10^{-2}[\mathrm{~Pa}]$ (see Figure 6) and $<1[\mathrm{~ms}]$ at atmospheric pressure $\sim 10^{5}[\mathrm{~Pa}]$. The simulated results are in good agreement with the measurements.

\section{Conclusions}

The thermal performance of a wafer level packaged MEMS thermal sensor based on CMOS-SOIMEMS technology and a suspended CMOS transistor has been simulated, modeled, and measured. 
The good agreement between the three approaches indicates the maturity and reproducibility of the technology. The overall performance of the sensing systems indicates why they are most suitable for consumer electronics, including smart homes, wearables, Internet of Things (IoT), and mobile applications.

Author Contributions: Conceptualization, Y.N.; methodology, Y.N., M.A., M.V., G.B. and M.E.C.; software, M.A.; validation, S.S. and T.B.; formal analysis, M.V.; investigation, M.A., S.S., M.V. and G.B.; resources, Y.N.; data curation, T.B.; writing - original draft preparation, M.A.; writing-review and editing, Y.N., S.S. and G.G.; visualization, M.A.; supervision, Y.N. and G.G.; project administration, Y.N.; funding acquisition, Y.N., M.V., G.B. and M.C. All authors have read and agreed to the published version of the manuscript.

Funding: The funding is provided by TODOS TECHNOLOGIES Ltd. (www.todos-technologies.com) which is the exclusive holder of the IP relating to this work.

Acknowledgments: The generous funding of TODOS TECHNOLOGIES Ltd. is gratefully aknowledged.

Conflicts of Interest: The authors declare no conflict of interest.

\section{References}

1. Blank, T.; Brouk, I.; Bar-Lev, S.; Amar, G.; Meimoun, E.; Meltsin, M.; Bouscher, S.; Vaiana, M.; Maierna, A.; Castagna, M.E.; Bruno, G.; Nemirovsky, Y. Non-Imaging Digital CMOS-SOI-MEMS Uncooled Passive Infra-Red Sensing Systems. IEEE Sens. 2020, doi:10.1109/JSEN.2020.3022095.

2. Zviagintsev, A.; Blank, T.; Brouk, I.; Bloom, I.; Nemirovsky, Y. Modeling the performance of nano machined CMOS transistors for uncooled IR sensing. IEEE Trans. Electron Devices 2017, 64, 4657-4663, doi:10.1109/TED.2017.2751681.

3. Nemirovsky, Y.; Stolyarova, S.; Blank, T.; Svetlitza, A.; Bar-Lev, S.; Zviagintsev, A.; Brouk, I. A new pellistor-like gas sensor based on micromachined CMOS transistor. IEEE Trans. Electron Devices 2018, 99 , 5494-5498, doi:10.1109/TED.2018.2878015.

4. Shlenkevitch, D.; Stolyarova, S.; Blank, T.; Brouk, I.; Nemirovsky, Y. Novel Miniature and Selective Combustion-Type CMOS Gas Sensor for Gas-Mixture Analysis-Part 1: Emphasis on Chemical Aspects. Micromachines 2020, 11, 345, doi:10.3390/mi11040345.

5. Avraham, M.; Stolyarova, S.; Blank, T.; Bar-Lev, S.; Golan, G.; Nemirovsky, Y. A Novel Miniature and Selective CMOS Gas Sensor for Gas Mixture Analysis-Part 2: Emphasis on Physical Aspects. Micromachines 2020, 11, 587, doi:10.3390/mi11060587.

6. Fraunhofer Institute for Ceramic Technologies and Systems IKTS. Available online: https://www.ikts.fraunhofer.de/en.html (accessed on 25 March 2020).

7. Azpeitia Urquia, M.; Allegato, G.; Paleari, S.; Tripodi, F.; Oggioni, L.; Garavaglia, M.; Nemirovsky, Y.; Blank, T. High vacuum wafer level packaging for uncooled infrared sensor. In Proceedings of the 2020 Symposium on Design, Test, Integration \& Packaging of MEMS and MOEMS (DTIP), Lyon, France, June 2020, doi:10.1109/DTIP51112.2020.9139148.

8. Budzier, H.; Gerlach, G. Thermal Infrared Sensors, Theory, Optimization and Practice; John Wiley \& Sons Ltd.: London, UK, 2011; ISBN978-0-470-87192-8.

9. ANSYS Mechanical. Available online: https://www.ansys.com/products/structures/ansys-mechanical (accessed on 1 October 2020).

10. Nemirovsky, Y.; Nemirovsky, A.; Melman, S. Gas Sensing Device Having Distributed Gas Sensing Elements and a Method for Sensing Gas. U.S. Patent 10,768,153, 8 September 2020.

11. Bergman, T.L.; Incropera, F.P.; DeWitt, D.P.; Lavine, A.S. Fundamentals of Heat and Mass Transfer; John Wiley \& Sons: London, UK, 2011; ISBN 978-0470-50197-9.

Publisher's Note: MDPI stays neutral with regard to jurisdictional claims in published maps and institutional affiliations.

(C) 2020 by the authors. Submitted for possible open access publication under the terms and conditions of the Creative Commons Attribution (CC BY) license (http://creativecommons.org/licenses/by/4.0/). 